\title{
EPILEPSIA: PERSONALIDAD, DEPRESIÓN, ATENCIÓN Y MEMORIA ${ }^{1}$
}

Diana Guerrero*, Yuli Infante*, Ximena Palacios-Espinosa**

\section{Resumen}

El objetivo principal de esta investigación fue identificar la presencia de alteraciones en la personalidad, el estado de ánimo (depresión), la atención y la memoria en 20 pacientes epilépticos, cuyas edades oscilaron entre 18 y 40 años. Esta evaluación se realizó utilizando la escala multidimensional abreviada del MMPI (minimult) para personalidad, el inventario de depresión de Beck (BDI) para depresión, el trail making test (TMT) para el nivel atencional y la escala de memoria de Weschler (WMS-III) para memoria. Los resultados obtenidos muestran ausencia de alteraciones clínicamente significativas de las dimensiones evaluadas. Las implicaciones de esta investigación se presentan en la discusión.

epilepsia, personalidad, psicosis, depresión, atención, memoria, trail making test, minimult, escala de memoria de Weschler (III), inventario de depresión de Beck.

EPI, epilepsia; DEP, depresión; minimult, escala multidimensional abreviada del MMPI; BDI, inventario de depresión de Beck; TMT, trail making test; WMS-III, escala de memoria de Weschler.

\section{Abstract}

The main purpose of this research was to identify the presence of personality, mood (depression), attention and memory disorders in 20 epileptic patients aged between 18 and 40 years. This evaluation was conducted with an abbreviated multidimensional scale of the Minnesota Multiphasic Personality Inventory [MMPI (Minimult)] to assess personality, Beck Depression Inventory (BDI) to assess depression, the Trail Making Test (TMT) to measure the level of attention and Wechsler Memory Scale (WMS-III) to assess memory. Results show no clinically significant alterations of the assessed issues. Implications of this research are stated in the discussion.

epilepsy, personality, psychosis, depression, attention, memory, Trail Making Test, Minimult, Wechsler Memory Scale (III), Beck Depression Inventory.

\section{Introducción}

Durante siglos la persona epiléptica ha sido estigmatizada, considerándola incluso discapacitada. Se teme por su vida y por las consecuencias que la enfermedad genere en sus diferentes áreas de ajuste y se le concibe como una persona enferma, débil y desprotegida, víctima de impredecibles ataques que comprometen su salud, su supervivencia y su funcionalidad social. De hecho, "a menudo, los problemas psicológicos y sociales a los que se enfrentan las personas epilépticas son más incapacitantes que las crisis mismas" ${ }^{1}$.
'Artículo basado en un trabajo de investigación realizado entre noviembre de 2003 y noviembre de 2005 en el servicio de neurología del Hospital de San José por investigadores de la Facultad de Psicología de la Universidad El Bosque, Bogotá, D.C., Colombia. 
de la memoria, fueron BDI, minimult, TMT formas A y B y WMS-III, respectivamente.

BDI: compuesto de 21 ítems (calificables de 0 a 3 puntos) que permiten apreciar el grado global de depresión, así como los cambios emocionales generales a lo largo del tiempo, reevaluar a la persona con la periodicidad que se estime oportuna y obtener la valoración precisa de las cuestiones que caracterizan típicamente a la depresión. ${ }^{20}$ Para la calificación e interpretación del BDI se tuvieron en cuenta las siguientes normas: 0-10, normal; 11-16, depresión leve; 17-20, depresión intermitente, es decir que tienen momentos de euforia y de pasividad total sin poder explicarla; 21-30, depresión moderada; 31-40, depresión grave; 40 o más, depresión aguda extrema o severa.

Minimult: permite medir rasgos psicopáticos y de la personalidad a través de ocho escalas clínicas: hipocondriasis, depresión, histeria, desviación psicopática, paranoia, psicastenia, esquizofrenia, hipomanía y cuatro escalas de validez: interrogación, alteración de respuestas, elementos severos de alteración clínica y tendencias a no reconocer o no entender la temática. ${ }^{21}$

TMT: prueba neuropsicológica dividida en dos partes que consiste en la realización de un trazado mediante un lápiz sobre una hoja que exhibe números (parte A) y luego otra que exhibe números y letras (parte B). La persona debe conectar en primer término los 25 números dispuestos en la hoja, haciéndolo del modo más veloz posible y sin levantar el lápiz de la hoja. El evaluador controla el tiempo que demora la persona en realizar el trazado y computa el rendimiento en segundos. Luego le entrega la parte B de la prueba, que consiste en la unión alternativa de un número y una letra, debiendo conservar tanto el orden los números (1-13) como el orden alfabético de las letras (A-L).

WMS-III: se utilizó la versión española para evaluar tanto la memoria inmediata como la de trabajo y la demorada. Cada uno de estos tipos de memoria se evalúa en dos modalidades (la auditiva y la visual) y con dos tipos de tareas (recuerdo y reconocimiento). Consta de once pruebas, seis principales y cinco opcionales. Las principales se aplican dos veces con un intervalo de alrededor de 30 minutos. ${ }^{22}$ En esta investigación se aplicaron las siguientes siete subpruebas.

Subtest I (información personal y actual): pregunta al paciente sobre su edad, fecha de nacimiento y la identificación de acontecimientos públicos actuales y recientes, tanto nacionales como locales.

Subtest II (orientación): formula preguntas sobre tiempo y lugar, incluyendo la situación actual del paciente.

Subtest III (control mental): estudia los automatismos (respuestas automáticas, como el alfabeto) y la realización de una secuencia conceptual sencilla (por ejemplo contar de cuatro en cuatro desde uno a 67).

Subtest IV (memoria lógica): evalúa el recuerdo inmediato de ideas verbales a partir de dos párrafos que son leídos al paciente. El libre, después de una presentación auditiva. El primer párrafo leído contiene 24 unidades de memoria o "ideas" y el segundo 22. El paciente recibe un punto por cada "idea" que recuerda, siendo la puntuación total el número de ideas recordadas en cada párrafo. Los adultos jóvenes (20-29 años) suelen obtener una media de nueve puntos por párrafo; los adultos mayores retienen un poco menos (cerca de ocho ideas por párrafo).

Subtest $V$ (dígitos): es similar al del WAIS-R pero difiere en que la versión del WAIS omite el ensayo de tres dígitos en la sección en orden directo y el ensayo de dos dígitos en la sección en orden inverso y no otorga crédito a los nueve dígitos en orden directo y ocho en inverso. La puntuación es igual que en el WAIS, dónde el número máximo de dígitos recordados en la secuencia más alta en ambas secciones se suma para obtener una puntuación total en dígitos.

Subtest VI (reproducción visual): es una tarea de dibujo de memoria visual inmediata. Consta de cuatro 
dibujos, cada uno de los cuales se muestra al paciente durante diez segundos, después, el paciente debe dibujar de memoria con la mayor exactitud que pueda. Es muy sensible para la lesión del hemisferio derecho.

Subtest VII (aprendizaje asociado): estudia la retención verbal. Consiste en diez pares de palabras, seis de las cuales forman asociaciones "fáciles", es decir, con significado y cuatro pares de palabras "difíciles" que no son sencillos de asociar. La lista se lee tres veces, con una prueba de memoria después de cada lectura. La puntuación más alta es 21 puntos. $^{22}$

\section{Procedimilento}

La presente investigación se desarrolló en cuatro fases: 1) revisión teórica, 2) selección de instrumentos, 3) consecución de la muestra y 4) aplicación, dentro del marco de los principios de la investigación con seres humanos.

A todos los participantes se les proporcionaron las mismas instrucciones previamente protocolizadas, indicando la identidad de los investigadores, el nombre de la institución educativa que estaba respaldando la investigación, el carácter voluntario de la participación y la confidencialidad de la información. Quienes manifestaron el deseo de participar firmaron el consentimiento informado, se diligenció una ficha de datos sociodemográficos y el tipo de EPI se revisó en la historia clínica de cada paciente para obtener información confiable. Luego se prosiguió a la aplicación individualizada de cada una de las pruebas en el siguiente orden: 1) BDI, 2) Minimult, 3) TMT partes A y B (Figura 1) y 4) WMS-III.

\section{Resultados}

Las Tablas 1 a 13 muestran los resultados obtenidos. Los puntajes de los participantes en WMS-III muestran que en todos los subtests los participantes se encontraron ubicados en un rango de normalidad. En la Figura 2 se presenta en proporciones la cantidad de participantes que se encuentran dentro y fuera de la media establecida para la población colombiana, indicando que el 59\% están dentro de los rangos de normalidad, lo que indica que pueden desarrollar cualquier actividad o tarea que involucre memoria de trabajo.

La Figura 3 muestra la proporción de participantes que está por encima de la media en cada uno de los subtest del WMS-III, indicando que existe mayor habilidad en la ejecución de algunos de ellos. Por ejemplo, en la reproducción visual el 24\% mostró fortalezas a la hora de realizar tareas que requieren reproducir dibujos y transcribir información utilizan-

\begin{tabular}{|c|c|}
\multicolumn{2}{|c|}{ Tabla I. Datos sociodemográficos de los participantes } \\
\hline Edad: & Media: 30,5 años \\
\hline Sexo: & $\begin{array}{c}\text { Femenino: } 12 \\
\text { Masculino: } 8\end{array}$ \\
\hline Estrato \\
socioeconómico:
\end{tabular}

Nota. $\mathrm{N}=20$ personas.

\begin{tabular}{|l|c|}
\hline \multicolumn{2}{|c|}{$\begin{array}{c}\text { Tabla 2. Relación del número de personas según el } \\
\text { tipo de epilepsia }\end{array}$} \\
\hline Crisis focales o parciales simples & $\begin{array}{c}\text { Número de } \\
\text { personas }\end{array}$ \\
\hline Crisis focales o parciales complejas & 6 \\
\hline Crisis generalizadas & 5 \\
\hline Epilepsia del lóbulo temporal & 6 \\
\hline Epilepsia del lóbulo frontal & 2 \\
\hline
\end{tabular}

Nota. $N=20$ personas.

\begin{tabular}{|l|c|}
\hline \multicolumn{2}{|c|}{ Tabla 3. Resultados obtenidos en el BDI } \\
Grado de depresión & $\begin{array}{c}\text { Número de } \\
\text { personas }\end{array}$ \\
\hline Leve & 6 \\
\hline Intermitente & 5 \\
\hline Grave & 2 \\
\hline Severa & 2 \\
\hline
\end{tabular}

Nota: Quince de los pacientes evaluados presentaron algún grado de DEP. 


\begin{tabular}{|c|c|l|}
\hline \multicolumn{2}{|c|}{$\begin{array}{c}\text { Tabla I I. Resultados obtenidos en la escala hipomanía del minimult } \\
\text { Número de personas }\end{array}$} & $\begin{array}{c}\text { Puntuación } \\
\text { Interpretación }\end{array}$ \\
\hline 5 & $5-14$ & $\begin{array}{l}\text { Actividad y energía características de personas mentalmente } \\
\text { sanas. }\end{array}$ \\
\hline 12 & $15-20$ & $\begin{array}{l}\text { Enérgicas, agradables, entusiastas, sociables, amables, } \\
\text { optimistas, con autoconfianza, independientes. }\end{array}$ \\
\hline 3 & $21-24$ & \\
\hline
\end{tabular}

\begin{tabular}{|c|c|c|}
\hline Número de personas & $\begin{array}{c}\text { Tabla 12. Resultados obtenidos en el TMT parte A } \\
\text { Tiempo de ejecución en } \\
\text { segundos }\end{array}$ & Interpretación \\
\hline 14 & 29 & Ejecución promedio \\
\hline 4 & $30-90$ & Ejecución deficiente \\
\hline 2 & $>90$ & Fallo \\
\hline
\end{tabular}

\begin{tabular}{|c|c|c|}
\hline Número de personas & Tiempo de ejecución & Interpretación \\
\hline 14 & $<75$ segundos & Ejecución promedio \\
\hline 5 & $>125$ segundos & Ejecución deficiente \\
\hline 1 & $>3$ minutos & Fallo \\
\hline
\end{tabular}

do su memoria de trabajo o inmediata.

\section{Discusión}

La tendencia a evaluar la calidad de vida en diversas poblaciones de personas sanas y enfermas es evidente desde las últimas dos décadas del siglo XX hasta la actualidad. Ello está determinado, entre muchos otros factores, por la aparición y posicionamiento de modelos más salutogénicos y menos biomédicos, que favorecen la percepción de bienestar subjetivo en los seres humanos, lo que ha desencadenado una serie de esfuerzos significativos por establecer mecanismos de acción y control para aumentar dicha percepción.

Los datos encontrados en este estudio no son ge- neralizables; solo se consolidan como un punto de partida para la reflexión sobre las características de las personas epilépticas a nivel de su personalidad, su estado de ánimo y sus capacidades cognoscitivas y sobre el tratamiento que deben recibir para favorecer su calidad de vida.

Sería interesante que en futuras investigaciones se considerara la variable tiempo de convivencia con la EPI, incluso a través de estudios comparativos en diferentes momentos, ya que según Hersen y Bellack y Maldonado, Fernández y Ruíz, la persona epiléptica posee mayores dificultades de adaptación social que un sujeto libre de la enfermedad. ${ }^{2,8}$

De acuerdo con la literatura, la EPI del lóbulo temporal es a la que más se asocian trastornos de personalidad, 


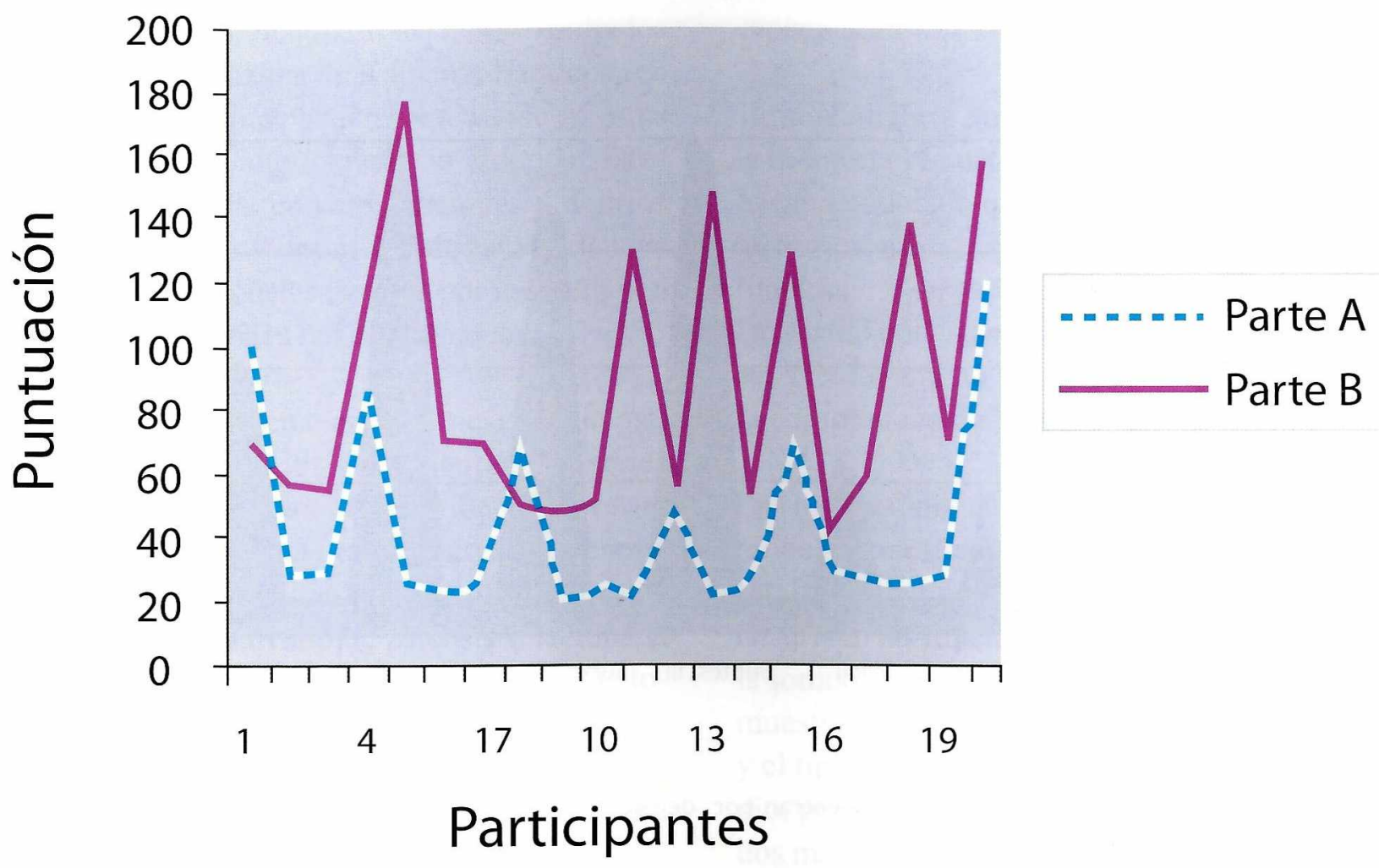

Figura I. Puntuaciones obtenidas por los sujetos en el TMT partes A y B.

psicosis y otras alteraciones del comportamiento. Afirmación que no se confirmó en los dos participantes con este tipo de EPI. Por supuesto, es una población insuficiente y no se pretende de ninguna manera generalizar este hallazgo. Solo es un indicador que permite establecer la necesidad de realizar investigaciones con una muestra representativa de pacientes con este tipo de EPI para evaluar y apoyar o contradecir la presencia de alteraciones comportamentales y cognoscitivas que describe la literatura.

Con respecto a la comorbilidad de la EPI con la depresión, se encontró que tal y como lo plantearon Marquez y Bortz, una proporción significativa de los participantes presentaban algún grado de depresión (75\% en el BDI y $55 \%$ en la escala de depresión del minimult). Se observó mayor prevalencia en los hombres. ${ }^{10,6}$

De acuerdo con los datos encontrados en este estudio y en la literatura, se recomienda que en futuras investiga- ciones se considere un control estricto de los fármacos consumidos por los pacientes epilépticos, de manera tal que se puedan establecer comparaciones entre unos y otros y así definir su posible influencia en el estado depresivo. De todas maneras los efectos psicológicos y sociales producidos por la enfermedad no pueden olvidarse. Se ha sugerido que el estigma asociado con la enfermedad y el prejuicio social contribuyen a los sentimientos depresivos de muchos enfermos y del mismo modo, la naturaleza impredecible de los ataques ha sido señalada como un factor importante. ${ }^{8}$

Con respecto a la personalidad, no hubo rasgos hipocondriacos entre los participantes, mientras que una proporción significativa (40\%), presentó rasgos histéricos. En cuanto a la escala esquizofrenia, ninguno de los sujetos mostró tener los rasgos completamente definidos. No obstante, se observó una leve tendencia al hecho de que son personas con poco interés en la gente y en asuntos prácticos. Son 


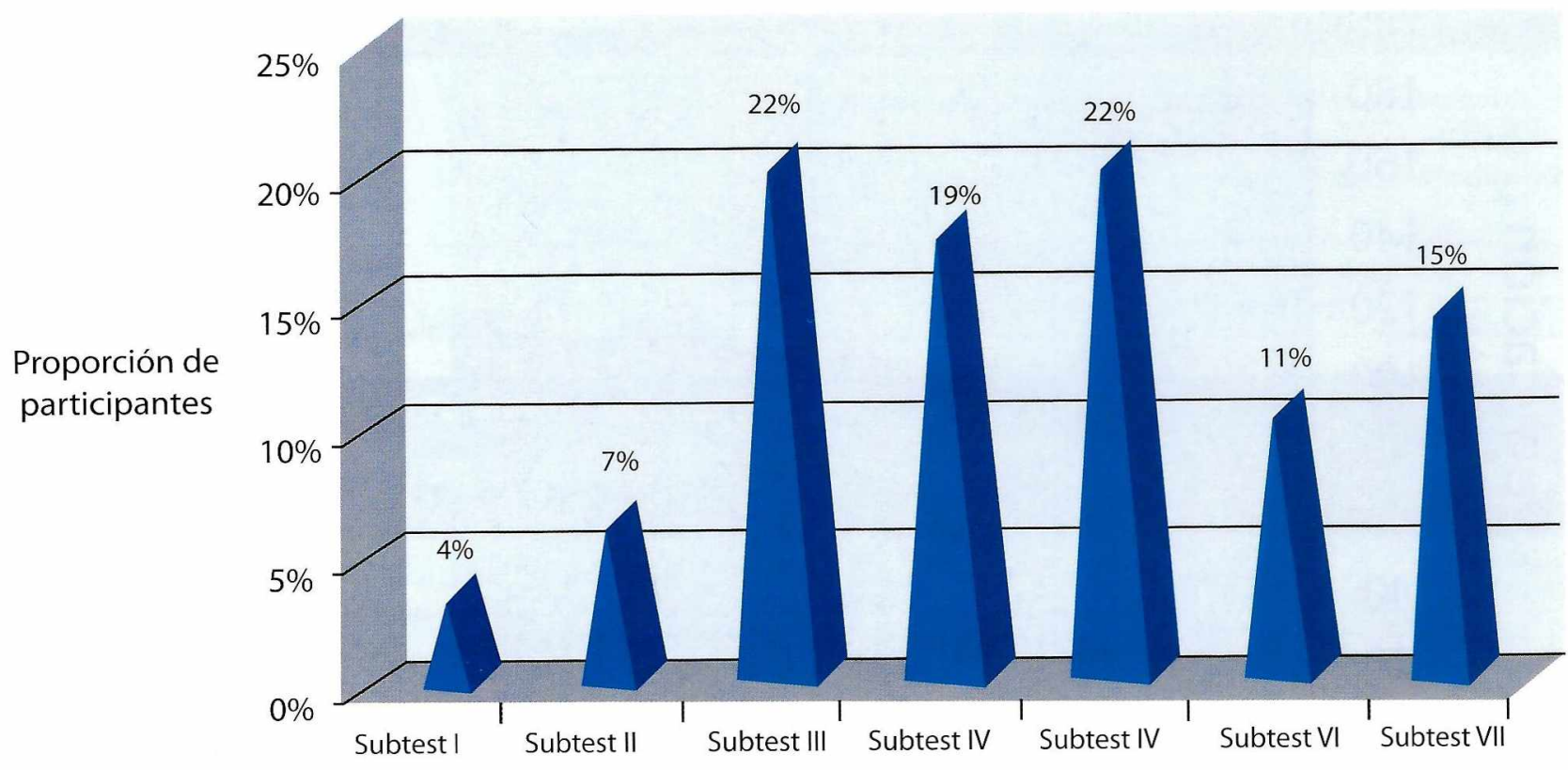

Figura 2. Proporción de participantes que se encuentran por debajo de la media en cada una de los subtest de la WMS-III.

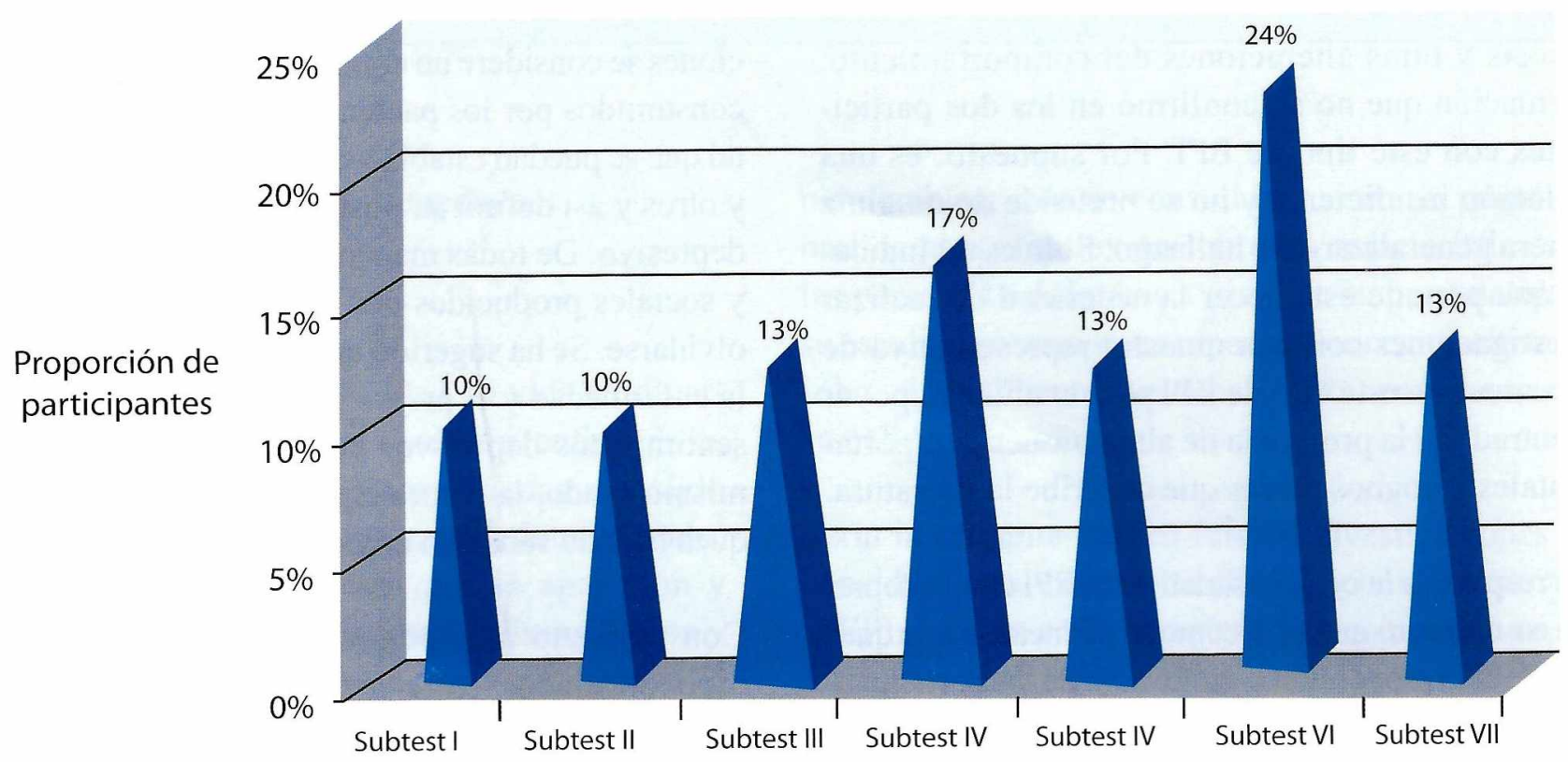

Figura 3. Proporción de participantes que se encuentran por encima de la media en cada una de los subtest de la WMS-III 
convencionales, aceptan la autoridad, tienen control de sí mismos y poco imaginativos. Estos resultados contradicen los hallazgos de Kaplan y Sandock, en los cuales reportan que pueden presentarse en estos pacientes episodios interictales con algunos síntomas de esquizofrenia, con alucinaciones y delirios paranoides que son los síntomas más característicos de esas psicosis, las cuales ocurren por lo regular en ausencia de alteraciones del estado de ánimo. ${ }^{5}$

Los estudios no han demostrado un exceso de psicosis en personas con EPI. Sin embargo, las series de casos clínicos indican con claridad que es un problema significativo en quienes acuden a centros especializados. Esto sugiere que existen factores de riesgo para el desarrollo de psicosis relacionada con EPI complicada y enfermedad crónica. ${ }^{5}$ De todas maneras, no puede ignorarse el planteamiento de Perrine y Conget con respecto a que los inicios de las psicosis a menudo ocurren después de una larga duración de la EPI (10 a 20 años).$^{13}$ Por ello, en futuros estudios también sería pertinente definir el tiempo de convivencia de estos pacientes con la enfermedad.

Con respecto a la atención, se encontró que más de la mitad de los participantes $(70 \%)$ tuvo niveles adecuados o promedio, mientras que el $30 \%$ restante bajos al momento de ejecutar la tarea, lo que podría estar relacionado con el tipo de EPI o con otras variables personales que en este estudio no se evaluaron. Además, estos resultados no apoyan el planteamiento realizado por Olaz según el cual hay evidentes alteraciones en la atención y la concentración de los pacientes epilépticos y más aún en aquellos con rasgos esquizoides, que tampoco fueron evidenciados en este estudio. ${ }^{23}$

En relación con la memoria, fueron evidentes las dificultades para dar respuestas automáticas tales como decir el alfabeto en español, contar de siete en siete y repetir dígitos en orden directo e inverso. Es posible que estos hallazgos estén ligados al nivel educativo. En futuros estudios podría considerarse establecer si se correlacionan con el nivel de atención, por ser este un componente central en el desarrollo de las capacidades mnésicas.

También habría que definir si estas deficiencias en la memoria se correlacionan con la depresión, pues es bien conocido que el paciente depresivo presenta compromiso cognoscitivo de origen afectivo no funcional, o en su defecto, podría también estar relacionado con los medicamentos antiepilépticos por sus efectos a nivel de atención y concentración y en la complejidad de la preservación mnésica.

Las limitaciones del estudio fueron el tamaño pequeño de la muestra, no considerar qué medicamentos estaban tomando pues es bien sabido que pueden interferir en las funciones cognoscitivas superiores por la somnolencia que provocan, no haber balanceado la muestra según las características sociodemográficas y el tipo de EPI, aunque no era un objetivo del estudio, pero es posible que hubiera favorecido resultados más concluyentes y la selección del instrumento para evaluar la personalidad. Aunque el minimult es una versión abreviada del Inventario de la Personalidad de Minnessota- MMPI, en realidad habría sido pertinente utilizar este último para obtener un perfil psicopatológico más significativo. No obstante, son bien conocidas las debilidades del MMPI en términos de extensión y tiempo de aplicación.

Dado que los resultados de este estudio no pueden generalizarse, no es posible establecer conclusiones definitivas. No obstante, esta primera aproximación resalta la contradicción con los hallazgos de estudios internacionales sobre la psicopatía y las alteraciones en la personalidad de los epilépticos. Aunque los resultados sugieren que hay mayor incidencia de alteraciones de la personalidad en las mujeres, se estima conveniente considerar la influencia de las variables socioculturales y la asunción del rol de enfermo tan particularmente reforzado en la sociedad colombiana, en especial en estratos socioculturales $\mathrm{y}$ en niveles educativos bajos.

La persona epiléptica puede gozar de altos niveles de calidad de vida siempre y cuando logre ajustarse 
y adaptarse a la situación crónica con la que con alta probabilidad vivirá de manera indefinida. ${ }^{25}$ Para ello, es necesario brindar tratamiento integral que incluya el apoyo neurológico y psicológico, este último dirigido a incrementar la competitividad social y el uso de estrategias de afrontamiento efectivas basadas en la solución de problemas y el control emocional.

Por consiguiente, una adecuada vigilancia y control de la evolución clínica de estas personas desde un marco multidisciplinario, podría favorecer la prevención del deterioro cognoscitivo y potencializar en ellos habilidades dirigidas a incrementar su calidad de vida.

\section{Referenclias}

1. Ivanovic-Zuvic F, Alvarado L. Evaluación psicosocial de los epilépticos en Chile. Rev Chil Neuropsiquiatr. 2001 Oct;39(4):303-315.

2. Hersen M, Bellack A, editors. Behavioral assessment: a practical handbook. Nueva York: Pergamon; 1976.

3. Geschwind N. Behavioural changes in temporal lobe epilepsy. Psychol Med. 1979 May;9(2):217-9.

4. Blume, WT. Motor system: anatomy, physiology, and epileptogenesis. En: Wyllie E. The treatment of epilepsy: principles \& practice. 2nd ed. Baltimore, Lippincott Williams \& Wilkins; 1996. p. 82-92.

5. Kaplan HI, Sadock BJ, Grebb JA. Compendio de psiquiatría. $7^{a}$ ed. Porto Alegre: Artes Médicas; 1997.

6. Bortz JJ. Neuropsychiatric and memory issues in epilepsy. Mayo Clin Proc. 2003 Jun;78(6):781-7.

7. Mendez MF, Cummings JL, Benson DF. Depression in epilepsy. Significance and phenomenology. Arch Neurol. 1986 Aug;43(8):766-70.

8. Maldonado J, Fernández F, Ruíz P. Depresión en pacientes que sufren desórdenes neurológicos. Alcmeón 1997;22(1):136-43.

9. Weissman MM, Klerman GL. Sex differences and the epidemiology of depression. Arch Gen Psychiatry. 1977 Jan;34(1):98-111.
10. Marquez R. Tratamiento natural de la depresión. Buenos Aires: Paidos; 1990.

11. Kanner AM, Barry JJ. Is the psychopathology of epilepsy different from that of nonepileptic patients? Epilepsy Behav. 2001 Jun;2(3):170-186.

12. Lambert MV, David AS. Psychiatric comorbidity. In: Oxbury JM, Polkey CE, Duchowny M, editors. Intractable focal epilepsy. Toronto: WB Saunders; 2000. p. 445-71.

13. Perrine K, Congett S. Neurobehavioral problems in epilepsy. Neurol Clin. 1994 Feb;12(1):129-52.

14. Toone BK. The psychoses of epilepsy. J Neurol Neurosurg Psychiatry. 2000 Jul;69(1):1-3.

15. Benson DF. The Geschwind syndrome. Adv Neurol. 1991;55:411-21.

16. Torta R, Keller R. Behavioral, psychotic, and anxiety disorders in epilepsy: etiology, clinical features, and therapeutic implications. Epilepsia. 1999;40 Suppl 10:S2-20.

17. Trevisol-Bittencourt PC, Troiano AR. [Interictal personality syndrome in non-dominant temporal lobe epilepsy: case report]. Arq Neuropsiquiatr. 2000 Jun;58(2B):548-55.

18. Dupont S, Samson Y, Van de Moortele PF, Samson S, Poline JB, Hasboun D, Le Bihan D, Baulac M. Bilateral hemispheric alteration of memory processes in right medial temporal lobe epilepsy. J Neurol Neurosurg Psychiatry. 2002 Nov;73(5):478-85.

19. Lopera, F. Amnesia tipo Korsakoff. En: Ardila A, Montañes P, Rosselli M. La memoria: principios neuropsicológicos. Medellín: Prensa Creativa; 1985. p. 107-17.

20. Téllez J, Taborda LC, Burgos C. Psicopatología clínica: el síntoma en las neurociencias. Bogotá: Fundación Universitaria de Ciencias de la Salud; 2000.

21. Kincannon JC. Prediction of the standard MMPI scale scores from 71 items: the mini-mult. J Consult Clin Psychol. 1968 Jun;32(3):319-25.

22. Wechsler D. A standardized memory scale for clinical use. J Psychol. 1945;19:87-95.

23. Tornimbeni S, Pérez E, Baldo M. Introducción a los test psiológicos. Córdoba: Brujas; 2000.

24. Pozo D, Pozo AJ. Nuevo enfoque conceptual de la epilepsia. Rev Cubana Pediatr. 2001 Oct;73(4):35-41.
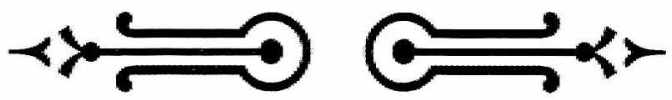